\title{
Educación Física en centros bilingües: efecto de la utilización del idioma sobre la percepción de la comunicación docente y la motivación del alumnado Physical Education in Bilingual Schools: Effect of the language use on students' perceptions of teaching communication and motivation
}

*Víctor Sánchez-Vegas, *Javier Coterón, *,**Alba González-Peño, **Evelia Franco

*Universidad Politécnica de Madrid (España), **Universidad Pontificia Comillas (España)

Resumen. En los últimos años es frecuente la impartición de la educación física (EF) en lengua inglesa, lo que puede influir sobre las percepciones que los alumnos ${ }^{1}$ tienen sobre dicha asignatura. En el presente estudio se analizó el efecto de una metodología bilingüe, el code-switching, sobre la percepción de la comunicación docente y la motivación. La muestra estuvo compuesta por 94 estudiantes de entre 12 y 14 años $(M=13.76$; $D T=.86)$. El grupo experimental $(\mathrm{n}=46)$ estuvo formado por dos grupos pertenecientes a formas diferentes de implantación del bilingüismo (programa y sección) y el grupo control $(\mathrm{n}=47)$ por dos grupos equivalentes a los anteriores. Previo a la intervención, se realizaron entrevistas a profesorado de EF; los cuestionarios pre y post fueron administrados al alumnado al inicio y al fin de la misma. Tras la intervención se hallaron diferencias en la motivación intrínseca de uno de los grupos experimentales. Los resultados sugieren que la percepción de la comunicación docente por parte de los alumnos no difiere en función de que la clase se imparta íntegramente en inglés o utilizando el code-switching. Se discuten las implicaciones educativas de estos hallazgos.

Palabras clave: educación física, bilingüismo, comunicación docente, motivación intrínseca.

Abstract. Over the last few years, the teaching of PE in English language has become frequent which could influence the perceptions that students have about this subject. In the present study the effect of a bilingual methodology, code-switching, on perceptions of teaching communication and motivation was analyzed. The sample was composed by 94 students aging from 12 to $14(M=13,76 ; S D=.86)$. Experimental group $(\mathrm{n}=46)$ was formed by two classes with different bilingual programs (programa and sección) and control group $(\mathrm{n}=47)$, by other two equivalent groups. After the intervention significant differences in intrinsic motivation were found in one of the experimental groups. The findings suggest that the perceptions of teaching communication do not differ between students taught completely in English and students taught through codeswitching. Educational implications of these findings are discussed.

Keywords: physical education, bilingualism, teaching communication, intrinsic motivation.

\section{Introducción}

En la última década la Comunidad Autónoma de Madrid (CAM) ha implantado progresivamente la enseñanza bilingüe en centros educativos no universitarios. En los institutos bilingües de educación secundaria la educación física (EF) es una de las materias que más frecuentemente ha sido seleccionada para su impartición en inglés.

A nivel estatal, en el año 1996 comenzó el desarrollo de proyectos curriculares integrados español-inglés en centros educativos públicos gracias al convenio firmado entre el Ministerio de Educación y Ciencia y The British Council. Una vez transferidas las competencias

Fecha recepción: 30-04-21. Fecha de aceptación: 16-07-21

Alba González-Peño

alba.gonzalez.peno@alumnos.upm.es educativas a las comunidades autónomas, la Comunidad Autónoma de Madrid puso en marcha el programa de colegios bilingües en el curso 2004/05, convirtiéndose desde entonces en uno de los ejes característicos de sus políticas educativas. Se ha pasado de contar con 26 centros educativos públicos bilingües de Primaria en el curso 2004/05 a 464, 354 de primaria y 110 de secundaria, en el curso 2015/16. Existen dos modalidades diferentes de impartición: sección bilingüe, en la que se cursan 5 horas semanales de inglés avanzado además de todas las materias en inglés excepto lengua y literatura, matemáticas y segunda lengua extranjera; y programa bilingüe, en el que se cursan 5 horas semanales de inglés además de cursar también en este idioma EF, educación plástica y visual, y música (Consejería de Educación, 2013). Como se puede observar, la EF es una de las asignaturas que se cursan en inglés independientemente de la modalidad en que se encuentre matriculado el alumnado.

Tomando como referencia el estudio realizado por 
el Ministerio de Educación y The British Council (Dobson, Pérez, y Johnstone, 2010) sobre las repercusiones que estos programas bilingües están ocasionando en el alumnado español, parecen obvios los beneficios que los mismos provocan tanto en el nivel curricular alcanzado por los participantes como en la percepción positiva que los agentes implicados tienen de ese tipo de estudios (Hernando, Hortigüela y Pérez-Pueyo, 2018). Sin embargo, son muchas las dudas surgidas dentro de la comunidad educativa en referencia a este tipo de programas (Pavón Vázquez y Rubio, 2010), destacando la carencia de metodología específica y contrastada en el conjunto de las materias (Halbach, 2008), especialmente en la EF (Coral i Mateu, 2013; Macaro, 2001; Manchunsky, 2008).

La enseñanza de idiomas ha ido variando con el paso de los años (Ramos y Ruiz, 2010), pero dentro de EF se ha implantado de forma generalizada el Aprendizaje Integrado de Contenido y Lengua (AICOLE o CLIL), entendiendo como tal una situación en la que el profesor ${ }^{1}$ de cualquier asignatura utiliza un idioma extranjero, en mayor o menor medida, como lengua vehicular de dicha materia (Coyle, Hood, y Marsh, 2010) no existiendo la necesidad, ni legal ni pedagógica, de impartir la totalidad de la unidad didáctica o de los contenidos en lengua extranjera (Molero Clavellinas, 2011). Sin embargo, la enseñanza descrita suele realizarse de forma monolingüe, utilizando exclusivamente el inglés como idioma de comunicación durante las clases (ChivaBartoll, Isidori, y Fazio, 2015)Esta inmersión directa en la lengua extranjera es cuestionada por diversos estudios (Gallagher y Colohan, 2017; Hornberger y Link, 2012a) que abogan por el uso conjunto de ambos idiomas en las primeras etapas de aprendizaje, adquiriendo mayor peso la lengua extranjera a medida que ésta se va dominando (Macaro, 2001). Varios autores consideran la no utilización de ambos lenguajes un sinsentido que lastra el abanico de recursos cognitivos, intelectuales y lingüísticos de los que disponen tanto los docentes como el alumnado (Butzkamm, 2007; Hornberger y Link, 2012b) en tanto que existen estudios neurolingǘsticos que demuestran cómo en el cerebro de las personas bilingües ambos idiomas permanecen activos y accesibles en todo momento independientemente de cuál de los dos esté siendo utilizado, y cómo aquellas personas que normalmente mezclan códigos o lenguas en su día a día, son capaces de producir un lenguaje totalmente monolingüe cuando hablan con una persona que sólo conoce uno de los dos idiomas (García y Beardsmore, 2008; Lewis, Jones, y Baker, 2012).
Entre los métodos utilizados para promover una enseñanza bilingüe, cabe destacar el code-switching (cambio de código), definido por Rotaetxe (1999) como «la alternancia en el uso de dos lenguas o variedades, distintas y reconocibles, bien por parte de un locutor, bien por parte de varios en un mismo discurso» (p.60). Aplicando esta definición al campo de la metodología de enseñanza, se define como la alternancia en el uso de dos lenguas en función de la actividad a desarrollar y del objetivo perseguido, mezclando en este caso el inglés y el castellano. Puesto que la comprensión y análisis de dichos conceptos y contenidos en profundidad por parte del alumnado es un requisito imprescindible para la asimilación de los mismos, la opción de utilizar el codeswitching permite el desarrollo y enriquecimiento a nivel de vocabulario y reglas gramaticales del aprendiz (Coyle et al., 2010; Lewis et al., 2012).

En el desarrollo de los programas bilingües el papel del profesor cobra una especial relevancia, dado que la utilización de una segunda lengua puede convertirse en una barrera para la correcta comunicación con los estudiantes y la comprensión por parte de estos de las consignas de trabajo (Jes y Molero, 2009; Molero Clavellinas, 2009). Además, el dominio del segundo idioma por parte del alumnado también va a jugar un papel determinante en su percepción de la asignatura, ya que la utilización de dicho idioma puede transformarse involuntariamente en un elemento extraño que afecte negativamente el interés de los alumnos por la EF (Freeman, Freeman, y Mercuri, 2005; Ramos y Ruiz, 2011). Aunque generalmente el docente emplea más tiempo en decidir qué va a transmitir que en cómo va a hacerlo (Moreno-Murcia, Huéscar, Peco, Alarcón, y Cervelló, 2012), las habilidades comunicativas juegan un papel esencial en este tipo de enseñanza en tanto que el desarrollo de las clases se lleva a cabo en una segunda lengua (Lova, 2013). En los últimos años ha aumentado el estudio de los procesos comunicativos como herramienta de mejora en el proceso enseñanza-aprendizaje (García Sánchez y Cruz Vargas, 2013;Viciana, Cervelló, y Ramírez-Lechuga, 2007; Viciana, Salinas, Ramírez, Cocca, y Cervelló, 2007), siendo escasos los estudios específicos encontrados en el ámbito de la EF (MorenoMurcia et al., 2012) Si bien la literatura no es muy prolija en este tipo de investigaciones, también hay estudios centrados en las consecuencias del uso de estrategias comunicativas en lengua extranjera. En esta línea, Alonso et al. (2015) sugirieron que el uso del inglés como lengua vehicular puede favorecer la motivación, implicación y deshinibición del alumnado en la clase de 
EF. Otros autores encontraron mayores niveles de atención en clase (Salvador-García, Chiva-Bartoll y CapellaPeris, 2020), así como un incremento de la participación del alumnado (Salvador-García, Chiva-Bartoll y Gallego, 2018). Sin embargo, Coral et al. (2020) hallaron un descenso del tiempo activo de los alumnos durante las clases de EF debido al uso de materiales adicionales para facilitar la comunicación en la lengua inglesa.

Una de las principales preocupaciones en la adquisición de una segunda lengua ha sido la percepción y la motivación del alumnado hacia el aprendizaje de este idioma (Baena Extremera y Granero-Gallegos, 2015; Khodadady y Khajavy, 2013; Rodríguez-Pérez, 2014), pues esta variable ha sido ampliamente aceptada por los docentes e investigadores como uno de los factores clave que influyen en el éxito del aprendizaje de une lengua extranjera (Ghazvini y Khajehpour, 2011). La motivación está estrechamente ligada a algunas de las teorías psicológicas más estudiadas en estos años en el aprendizaje como es la teoría de la autodeterminación (Khodadady y Khajavy, 2013) o el modelo multidimensional de la motivación de logro (Ruiz Pérez, Moreno Murcia, Ramón Otero, y Alias García, 2015).

La teoría de la autodeterminación (TAD; Deci y Ryan, 2000) es una macro-teoría cuyo objeto de estudio es la motivación humana y que trata de explicar el desarrollo de la personalidad dentro de contextos sociales. Esta teoría se ha desarrollado durante las últimas décadas a través de seis mini-teorías. La teoría de la integración orgánica establece un continuo de autodeterminación que va desde la desmotivación hasta la motivación intrínseca. Ésta última se define como el placer que se experimenta al realizar una actividad sin recibir una gratificación externa directa (Csikszentmihalyi y Rathunde, 1993; Ryan, 1995). El desarrollo de la actividad es el fin en sí mismo, generando sensaciones de competencia y autorrealización. El análisis de la motivación intrínseca en EF se ha abordado, en su mayor parte, desde trabajos de corte transversal en los que se describen relaciones con otras variables que demuestran ser antecedentes de la aparición de la motivación intrínseca o consecuencias de la misma. Se han encontrado relaciones positivas con distintas variables presentes en el contexto de EF, como orientación a la tarea (Cañabate et al., 2014; GraneroGallegos y Baena-Extremera, 2014), intención futura de ser físicamente activos (Franco et al., 2017), actitud cognitiva y afectiva (Sevil et al., 2016) o comportamientos y actitudes positivas (Sánchez-Oliva, 2014). .
Sin embargo, no se han encontrado trabajos relacionados con el objeto de nuestro estudio.

El modelo multidimensional de motivación de logro para el aprendizaje en contextos de EF (Nishida, 1989) considera que las personas manifiestan una motivación genérica hacia el logro y que ésta se expresará en el aprendizaje en las clases de EF, abarcando la disposición emocional del individuo, la conciencia del valor que da a lo que aprende, la autoevaluación de su propia competencia, así como la influencia de experiencias pasadas de éxito o fracaso. Factores como el compromiso con la práctica, la valoración del aprendizaje, la competencia motriz percibida o el miedo al error son elementos que determinan la motivación de los alumnos hacia la educación física (Ruiz Pérez et al., 2015). De esta forma, los alumnos más motivados para el logro conseguirán un mayor aprendizaje en habilidades motrices que aquellos que muestran una motivación para evitar el fracaso.

No se han encontrado estudios que analicen si el hecho de recibir las clases de EF en una lengua extranjera pueda influir sobre determinados aspectos de la relación que el alumnado establezca con la asignatura. El objetivo de nuestro trabajo fue analizar el efecto de una metodología bilingüe, frente a una monolingüe, sobre la percepción de la comunicación docente, la motivación intrínseca, competencia autopercibida y comparada, ansiedad ante el fracaso y compromiso con el aprendizaje en estudiantes de EF.

\section{Metodología}

\section{Participantes}

La muestra estuvo formada por 94 estudiantes (49 chicos y 45 chicas) pertenecientes a cuatro grupos de $2^{\circ}$ de educación secundaria obligatoria de un instituto público de la CAM con edades comprendidas entre los 13 y 16 años $(M=13.76$ años; $D T=.86)$. La muestra se dividió en condición experimental $(n=46)$ y de control $(n=47)$, constando cada una de ellas de una clase de sección $\left(\mathrm{n}_{\exp }=19, \mathrm{n}_{\text {cont }}=22\right)$ y otra de programa $\left(\mathrm{n}_{\exp }=27\right.$ , $\left.\mathrm{n}_{\text {cont }}=25\right)$.

\section{Materiales $e$ instrumentos}

Cuestionario de Motivación de Logro para Aprender en Educación Física.

Se utilizó la nueva versión adaptada al contexto español (Ruiz Pérez et al., 2015) de la anterior versión española del Cuestionario AMPET (Ruiz Pérez, Graupera, Gutiérrez, y Nishida, 2004) formada por un 
total de 32 ítems agrupados y divididos en cuatro dimensiones, que son la competencia motriz autopercibida (nueve ítems) (ej. «Hasta el momento soy bueno/a en Educación Física sin realmente esforzarme en serlo»), la competencia motriz comparada (cinco ítems) (ej. «Siempre me he considerado de los/as mejores en Educación Física), el compromiso con el aprendizaje (nueve ítems) (ej. «Normalmente escucho las cosas que me dice mi profesor/a de Educación Física») y la ansiedad y agobio ante el fracaso (nueve ítems) (ej. «A menudo me pongo nervioso/a cuando practico los ejercicios en público»), que se responden en una escala tipo Likert que oscila entre 1 (totalmente en desacuerdo) y 5 (totalmente de acuerdo). Los coeficientes alfa de Cronbach obtenidos en este estudio en las tomas pretest y postest fueron de .89 y .87 para competencia autopercibida, .86 y .85 para competencia comparada, .86 y .78 para compromiso con el aprendizaje, y .92 y .94 para la ansiedad y agobio ante el fracaso.

Escala de Evitación de Barreras de Comunicación en Educación Física (EEBCEF).

Se utilizó el cuestionario validado por Moreno-Murcia et al. (2012), compuesto de 12 ítems (ej. «No me interrumpe cuando hablo con él») y encabezado por el enunciado «El docente en clases de Educación Física...», que se responden en una escala tipo Likert que oscila entre 1 (totalmente en desacuerdo) y 5 (totalmente de acuerdo). Los coeficientes alfa de Cronbach obtenidos en este estudio en las tomas pretest y postest fueron de .93 y .86.

Escala del Locus Percibido de Causalidad en Educación Fisica (PLOC).

Se utilizó el factor motivación intrínseca de la Escala del Locus Percibido de Causalidad (PLOC) de Goudas, Biddle, and Fox (1994) validada al contexto español por Moreno, González-Cutre, and Chillón (2009). Está compuesto por un total de cuatro ítems (Ej. «Porque la Educación Física es divertida) encabezados por el enunciado «Participo en esta clase de Educación Física...». Se responden mediante una escala tipo Likert de 1 (totalmente en desacuerdo) a 5 (totalmente de acuerdo). Los coeficientes alfa de Cronbach obtenidos en este estudio en las tomas pretest y postest fueron de .91 y .89 .

\section{Diseño y procedimiento}

Se utilizó un diseño cuasi-experimental con grupo control no equivalente (Thomas y Nelson, 2007), puesto que al estar los grupos de clase ya establecidos por el centro de enseñanza no se podía respetar la aleatorización, si bien sí fue aleatoria la asignación de cada uno de ellos a la condición control y experimental.

Previo al estudio, se realizaron dos entrevistas a docentes especializados en EF y en lengua inglesa, con el objeto de recabar información de relevancia que pudiese mejorar el diseño y facilitar la comprensión de los resultados obtenidos (Coral i Mateu y Lleixà Arribas, 2013). Las dos semanas anteriores al inicio de la intervención se comenzó a aplicar todo el procedimiento a modo de semanas piloto de manera que las novedades percibidas por el alumnado no fueran tales una vez comenzase la recogida real de datos.

La intervención tuvo una duración de doce semanas entre los meses de enero y abril de 2015. El instituto donde se realizó el estudio contaba con cuatro grupos de $2^{\circ} \mathrm{ESO}$ con el mismo docente de EF, que recibían dos sesiones de EF semanales de 55 minutos de duración. Se obtuvieron los permisos y los consentimientos pertinentes conforme las indicaciones de los principios éticos y códigos de conducta de la Association (2002) y tanto antes de que comenzase la intervención como al concluir la misma, un colaborador externo formado a tal efecto administró al alumnado los cuestionarios descritos en el siguiente apartado.

Durante las doce semanas que duró la intervención los grupos control recibieron todas las sesiones de EF íntegramente en inglés, mientras que en los experimentales las sesiones fueron de naturaleza bilingüe utilizando el code-switching. De ese modo, en las clases impartidas a los grupos experimentales se mezclaba de manera consecutiva la comunicación en inglés-castellano, castellano-inglés. Se trabajaron dos Unidades Didácticas iguales para todos los grupos (una de fuerza integrada dentro de Judo, y una de habilidades gimnásticas integrada dentro de Parkour). Se organizaron dos grupos control, uno de Sección y uno de Programa, y dos grupos experimentales, uno de Sección y uno de Programa, garantizando así que el nivel inicial de inglés tanto en los grupos control como en los grupos experimentales fuese similar (ver Tabla 1). Las sesiones fueron grabadas en video (GoPro Hero3+) y audio (Voice Memos para iPhone) y analizadas con por dos observadores externos entrenados en el manejo del software Nvivo QSR International. Se utilizó un sistema de categorías diseñado ad-hoc con una fiabilidad del índice de Kappa de .86 en los que se recogieron los porcentajes observados de empleo de code-switching en cada una de las categorías (Tabla 2).

\section{Análisis de datos}

Se verificó la distribución de la muestra en las varia- 
bles de estudio a partir del test de Kolmogorov-Smirnov y se encontró la necesidad de realizar pruebas no paramétricas $(p<.05)$. A continuación, se llevó a cabo un test no paramétrico para muestras independientes (Kruskal-Wallis) para determinar si existían diferencias a priori entre los grupos. Tras la intervención se realizaron cuatro test no paramétricos de muestras relacionadas (Wilcoxon) para establecer diferencias intragrupo y otro test para muestras independientes (KruskalWallis) para analizar las diferencias entre grupos. Todos los análisis se realizaron con SPSS 20.0.

Tabla 1.

Distribución de grupos y contenido desarrollado durante la intervención

\begin{tabular}{ccccc}
\hline $\begin{array}{c}\text { GC Programa } \\
(\mathrm{N}=25)\end{array}$ & $\begin{array}{c}\text { GE Programa } \\
(\mathrm{N}=27)\end{array}$ & $\begin{array}{c}\text { GC Sección } \\
(\mathrm{N}=22)\end{array}$ & $\begin{array}{c}\text { GE Sección } \\
(\mathrm{N}=19)\end{array}$ \\
\hline Contenido & Fuerza aplicada al Judo \\
& \multicolumn{3}{c}{ Habilidades gimnásticas aplicadas al Parkour }
\end{tabular}

Metodología Monolingüe Monolingüe Code-switching Code-switching

Tabla 2.

Distribución de porcentajes de aparición de expresiones utilizando el code-switching

\begin{tabular}{lc}
\hline Categoría & Porcentaje \\
\hline Expresiones de bienvenida / despedida & $19.5 \%$
\end{tabular}

\begin{tabular}{ll}
\hline Expresiones de bienvenida / despedida & $19.5 \%$ \\
\hline
\end{tabular}

Instrucciones / Ordenes $\quad 12.9 \%$

Explicacion de conceptos

Feed-back positiv

Feed-back negativ

Total

$9.3 \%$

$9.3 \%$

$2.4 \%$

$100 \%$

\section{Resultados}

\section{Análisis preliminar}

El test no paramétrico para muestras independientes no mostró diferencias significativas en comunicación docente $\left(\div^{2}(3)=1.03, p^{>} .01\right)$, motivación intrínseca $\left(\dot{\div}^{2}(3)=2.01, p>.01\right)$, competencia autopercibida $\left(\div^{2}(3)=1.88, p^{>} .01\right)$, competencia comparada $\left(\div^{2}\right.$ $\left.(3)=1.88, p^{>} .01\right)$, compromiso con el aprendizaje $\left(\div^{2}\right.$ (3) $\left.=2.31, p^{>} .01\right)$ y ansiedad y agobio ante el fracaso $\left(\div^{2}(3)=3.25, p>.01\right)$.

\section{Análisis de la intervención}

La Tabla 3 muestra los resultados para cada variable dependiente antes y después de la intervención. No se observan diferencias en los dos grupos control ni en el

Tabla 3. $\frac{\text { Estadísticos descriptivos de las variables en las dos mediciones realizadas y diferencia de medias }}{\text { GC Programa } \quad \text { GE Programa Gección Ge Seccón }}$

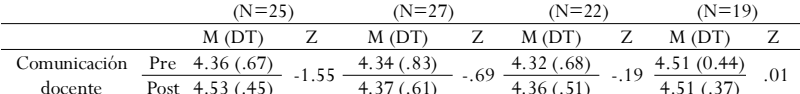

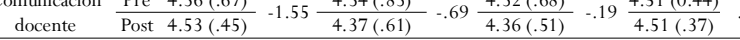
Motivación $\frac{\text { Pre 4.14(1.07) }}{n}-1.27 \frac{4.16(1.04)}{4.28(.77)}-.41 \frac{4.41(.62)}{4.28(.66)}-1.54 \frac{4.47(.93)}{4.14(1.10)}-2.14 *$

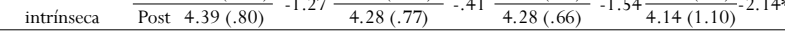

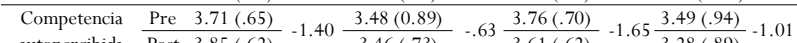

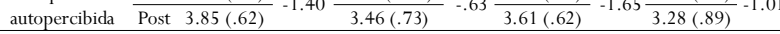

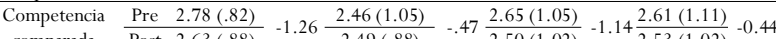

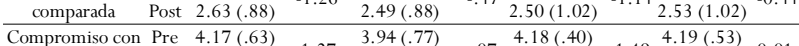

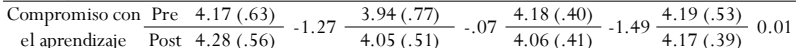
\begin{tabular}{crrrr} 
el aprendizaje & Post 4.28(.56) & $4.05(.51)$ & $4.06(.41)$ & $4.17(.39)$ \\
\hline Ansiedad y arobio & Pre $2.31(1.09)$ & $2.52(1.06)$ & $2.05(.87)$ & $2.05(.85)$
\end{tabular} Ansiedad
ante el fracaso grupo experimental de Programa. En el grupo experimental de Sección se encontró una diferencia significativa en la variable de motivación intrínseca $(\mathrm{p}<.05)$ siendo la puntuación mayor antes de la intervención que después.

\section{Diferencias entre los grupos tras la interven-} ción

En el análisis de comparación de puntuaciones tras la intervención no se encontraron diferencias significativas en ninguna de las variables; comunicación docente $\left(\div^{2}(3)=1.54, p^{>} .01\right)$, motivación intrínseca $\left(\div^{2}\right.$ (3) $=1.26, p>.01)$, competencia autopercibida $\left(\div^{2}\right.$ (3) $=6.09, p>.01)$, competencia comparada $\left(\div^{2}(3)=.26\right.$, $p>.01)$, compromiso con el aprendizaje $\left(\div^{2}(3)=5.99\right.$, $p>.01)$ y ansiedad y agobio ante el fracaso $\left(\div^{2}(3)=2.78\right.$, $p>.01)$.

\section{Discusión}

El objetivo de este trabajo fue analizar el efecto de una metodología bilingüe, frente a una monolingüe, sobre la percepción de la comunicación docente, la motivación intrínseca, competencia autopercibida y comparada, ansiedad ante el fracaso y compromiso con el aprendizaje en estudiantes de EF.

Al tratarse de una línea de investigación reciente, Salvador et al. (2017) realizaron una revisión de la literatura existente hasta el momento sobre el aprendizaje de una lengua extranjera en EF, encontrando que esta materia es capaz de aportar beneficios a distintos niveles como la mejora de las habilidades comunicativas y comprensivas del alumnado, el desarrollo de propuestas integradoras de la lengua por parte de los docentes, o la motivación de los estudiantes generada por estas propuestas desarrolladas en las clases.

En el presente estudio, de todas las variables analizadas, al comparar el efecto de una metodología bilingüe frente a una monolingüe, solo se detectaron diferencias significativas en la variable motivación intrínseca en el grupo experimental de Sección, habiendo disminuido al finalizar la intervención. En el resto de las variables no existieron diferencias significativas entre los grupos, ni antes ni después de la intervención, ni tampoco hubo variaciones intragrupo a lo largo de la misma. Pese a pertenecer a opciones diferentes dentro del programa de institutos bilingües, los cuatro grupos que formaron parte del estudio resultaron tener unas características bastante similares en relación a las variables analizadas, ofreciendo así unos resulta- 
dos homogéneos. Estos datos parecen indicar que el hecho de que el alumnado reciba las sesiones de EF íntegramente en inglés o utilizando code-switching no tiene un efecto directo sobre las variables analizadas. Esta afirmación nos hace pensar que las propias características de la materia son un aliciente claro para poder impartirla en una segunda lengua sin que dichos factores afecten al alumnado (Ramos y Ruiz, 2011).

No se encontraron diferencias significativas en la variable comunicación docente. Las altas puntuaciones observadas indican que ninguno de los grupos presentó dificultades a la hora de comprender las explicaciones del docente y seguir sus instrucciones, independientemente de cual fuese el idioma utilizado. Este hallazgo sugiere que, si bien es importante la comprensión del mensaje transmitido en la evitación de barreras de comunicación (Arellano, 2006), puede ser mayor la influencia de otros factores como la forma de escuchar con atención lo que dice el estudiante (Echeverría, 2017; Elizondo Torres, 1999; Marroquín, 1984) reflejada en la actitud respetuosa del docente, la empatía o el interés hacia el estudiante, o el tipo de feedback administrado por el docente (Moreno-Murcia, Huéscar, Peco, Alarcón, y Cervelló, 2013).

Como se ha indicado anteriormente, se encontraron diferencias significativas en la motivación intrínseca en el grupo experimental de Sección, decreciendo tras la realización de la intervención; pero los datos obtenidos no nos permiten afirmar categóricamente razones o consecuencias previsibles, no habiéndose encontrado literatura que pueda reforzar este hecho. En el resto de los grupos no se encontraron diferencias significativas para esta variable. Cabe mencionar que el grupo experimental de Sección ha recibido siempre sus clases íntegramente en inglés. El cambio a la enseñanza bilingüe planteada en la intervención, junto al hecho de que los sujetos pertenecientes a este grupo tengan una competencia relativamente alta en esta lengua extranjera, ha podido influir en los resultados observados. Tal y como postula la teoría de la evaluación cognitiva, el grado de desafío óptimo ayudará a lograr la motivación intrínseca (Deci y Ryan, 2000) por lo que el descenso observado en esta variable podría ser debido a que los individuos percibiesen que el reto que le suponía la nueva situación estuviese por debajo de sus capacidades.

No se encontraron diferencias significativas en la competencia autopercibida ni en la competencia comparada. La noción de competencia percibida hace referencia al nivel de éxito que normalmente el sujeto posee al interactuar con su medio (Gómez García, 2004) y la comparada se evalúa en relación a su grupo de iguales. La necesidad de sentirnos competentes a la hora de realizar las tareas y su relación con la motivación del individuo ha sido estudiada por numerosos autores (e.g., Deci y Ryan, 2000; Gutiérrez y Escartí, 2006) concluyendo que existe una relación positiva entre el nivel de competencia y la motivación intrínseca. En ese sentido, resulta de interés que las diferencias significativas señaladas anteriormente en el grupo experimental de Sección en relación a la motivación intrínseca no se vean acompañadas por un descenso en la percepción de su competencia, lo que podría sugerir la influencia de otros factores no considerados.

No se encontraron diferencias significativas en la variable compromiso con el aprendizaje. Pese a la importancia que este aspecto parece tener a priori el contexto educativo, la mayoría de las investigaciones en este campo se han realizado en el ámbito del deporte de competición, destacando la importancia del papel del entrenador en el compromiso de los deportistas (Almagro Torres, Sáenz-López Buñuel, González-Cutre Coll, y Moreno Murcia, 2011; Lukwu \& Guzmán Luján, 2011) o estudiando el estilo de comunicación (Torregrosa, Sousa, Viladrich, Villamarín, y Cruz, 2008). Tampoco se encontraron diferencias significativas en la variable ansiedad y agobio ante el fracaso. En este sentido, existen estudios (e.g., Cavazos Rehg, DeLuciaWaack, y Development, 2009) que analizaron el nivel de autoestima y ansiedad en centros bilingües y no bilingües, siendo ambas superiores en los centros bilingües. Sin embargo, no se ha encontrado bibliografía que relacione estas variables de manera directa con la EF en centros bilingües.

Si bien es cierto que con un tamaño de muestra reducido como el de la presente investigación resulta complicado extrapolar los resultados de forma genérica, el estudio ha aportado conclusiones de interés a la literatura existente. Considerando todo lo anterior, planteamos como futuras líneas de investigación, por un lado, el desarrollo de intervenciones de larga duración, así como la inclusión de enfoques cualitativos mediante entrevistas personales y/o grupos de discusión con el alumnado que pudiesen aportar información relevante que ayude a comprender de primera mano la vivencia que de la asignatura tienen quienes son sus principales protagonistas.

A nivel institucional sólo existe el estudio anteriormente mencionado de Dobson et al. (2010) sobre las repercusiones que este tipo de programas está teniendo sobre el alumnado, con la peculiaridad de que los 
resultados no quedan especificados por asignaturas. Resultaría de gran interés el poder conocer cómo se está viendo afectada la transmisión de los contenidos de EF y realizar una comparativa entre centros bilingües y no bilingües. En esta línea, resultaría interesante comprobar si el uso del code-switching puede tener efectos diferentes según los contenidos impartidos. Además, se plantea el desarrollo de programas de intervención para ofrecer a los docentes herramientas que les permitan saber cuándo utilizar la lengua nativa o extranjera al hacer uso del code-switching.

Por otro lado, dado que hay evidencias de la existencia de diferentes perfiles motivacionales entre el alumnado, se plantea el análisis de las posibles diferencias en la percepción de estrategias de comunicación referentes al bilingüismo según su perfil de motivación. Asimismo, sería interesante analizar cómo puede afectar el estilo interpersonal del docente, y su capacidad comunicativa y lingüística, sobre el desarrollo de sus clases y la percepción que tienen los alumnos al respecto. A modo de conclusión, se puede intuir que la utilización de una metodología bilingüe o monolingüe parece no tener una influencia determinante en las diferentes variables analizadas. Ante la ausencia de investigaciones específicas sobre EF y bilingüismo se hace necesario realizar estudios que vayan más allá de la elaboración de materiales didácticos con el fin de generar una base metodológica fundamentada en dichos hallazgos que pueda facilitar la labor docente.

\section{Agradecimientos}

El trabajo fue financiado por la Universidad Pontificia Comillas (PP2021_03) y la Agencia Estatal de Investigación española (PID2020-114051RA-I00 / AEI /10.13039/501100011033).

\section{Referencias}

Almagro Torres, B. J., Sáenz-López Buñuel, P. S.-L., González-Cutre Coll, D., \& Moreno Murcia, J. A. M. (2011). Clima motivacional percibido, necesidades psicológicas y motivación intrínseca como predictores del compromiso deportivo en adolescentes. RICYDE. Revista Internacional de Ciencias del Deporte, 7(25), 250-265.

Alonso, J. A., Cachón, J., Castro, R., \& Zagalaz, M. L. (2015). Propuesta didáctica bilingüe para educación física en educación primaria. Juegos populares y tradicionales ingleses. Retos, 28, 116-121.
Arellano, N. (2006). Las barreras en la comunicación no verbal entre docente-alumno. Orbis. Revista Científica Ciencias Humanas, 2(4), 3-38.

Association, A. P. (2002). Ethical Principles of Psychologists and Code of Conduct. American Psychologist, 57(12), 1060-1073. doi:10.1037/0003066X.57.12.1060

Baena Extremera,A., \& Granero-Gallegos, A. G. (2015). Versión española del Sport Satisfaction Instrument (SSI) adaptado al aprendizaje de la Educación Física bilingüe en Inglés. Porta Linguarum(24), 63-76.

Butzkamm, W. (2007). Native language skills as a foundation for foreign language learning. Trascending Boundaries(87), 71-85.

Cañabate, D., Torralba, J. P., Cachón, J., \& Zagalaz, M. L. (2014). Perfiles motivacionales en las sesiones de educación física. Retos, 26, 34-39.

Cavazos Rehg, P. A., DeLucia Waack, J. L., \& Development.(2009). Education, ethnic identity, and acculturation as predictors of self esteem in Latino adolescents. Journal of Counseling \& Development, 87(1), 47-54.

Chiva-Bartoll, O., Isidori, E., \& Fazio, A. (2015). Educación Física bilingüe y pedagogía crítica: una aplicación basada en el Judo. Retos, 28, 110-115.

Coral, J., Urbiola, M., Sabaté, E., Bofill, J., Lleixà, T., \& Vilà Baños, R. (2020). Does the teaching of physical education in a foreign language jeopardise children's physical activity time? A pilot study. International Journal of Bilingual Education and Bilingualism, 23(8), 839-854.

Coral i Mateu, J. (2013). Physical education and English integrated learning: How school teachers can develop PE-in-CLIL programmes. Temps d'Educació(45), 41-64.

Coral i Mateu, J., \& Lleixà Arribas, T. (2013). Las tareas en el aprendizaje integrado de educación física y lengua extranjera (AICLE). Determinación de las características de las tareas mediante el análisis del diario de clase. Retos: nuevas tendencias en educación física, deporte y recreación(24), 79-84.

Coyle, D., Hood, P., \& Marsh, D. (2010). CLIL: Content and language integrated learning. Cambridge: Cambridge University Press.

Csikszentmihalyi, M., \& Rathunde, K. (1993). The measurement of flow in everyday life: Toward a theory of emergent motivation.

Deci, E. L., \& Ryan, R. M. (2000). Self-determination theory and the facilitation of intrinsic motivation, social development, and well-being. American 
Psychologist, 55(1), 68-78.

Dobson, A., Pérez, M. D., \& Johnstone, R. (2010). Programa de educación bilingüe en España. Informe de la evaluación. Madrid: Ministerio de Educación. Gobierno de España.

Echeverría, R. (2017). Ontología del lenguaje (J. C. Sáez Ed.). Santiago: Ediciones Granica SA.

Elizondo Torres, M. (1999). Asertividad y escucha activa en el ámbito académico. Sevilla: Trillas.

Franco, E., Coterón, J., Gómez,V., Brito, J., \& MartínezGonzález, A. (2017). Influencia de la motivación y del flow disposicional sobre la intención de realizar actividad físico-deportiva en adolescentes de cuatro países. Retos, 31, 46-51.

Freeman, Y. S., Freeman, D. E., \& Mercuri, S. (2005). Dual language essentials for teachers and administrators. Porstmout, NH: Heinemann.

Gallagher, F., \& Colohan, G. (2017).T(w)o and fro: using the L1 as a language teaching tool in the CLIL classroom. The Language Learning Journal, 45(4), 485 498.

García, O., \& Beardsmore, H. B. (2008). Bilingual Education in the 21st Century: A Global Perspective. Oxford: Wiley-Blackwell.

García Sánchez, M. E., \& Cruz Vargas, M. L. (2013). Factores motivacionales extrínsecos e intrínsecos en el aula de inglés: análisis empírico. Porta Linguarum(19), 275-298.

Ghazvini, S. D., \& Khajehpour, M. (2011). Attitudes and Motivation in learning English as Second Language in high school students. Procedia Social and Behavioral Sciences, 15, 1209-1213.

Gómez García, M. (2004). Problemas evolutivos de coordinación motriz y percepción de competencia en el alumnado de primer curso de educación secundaria obligatoria en la clase de educación física: Universidad Complutense de Madrid.

Goudas, M., Biddle, S., \& Fox, K. (1994). Perceived locus of causality, goal orientations, and perceived competence in school physical education classes. British Journal of Educational Psychology, 64, 453-463.

Granero-Gallegos, A., \& Baena-Extremera, A. (2014). Predicción de la motivación autodeterminada según las orientaciones de meta y el clima motivacional en Educación Física. Retos, 25, 23-27.

Gutiérrez, M., \& Escartí, A. (2006). Influencia de padres y profesores sobre las orientaciones de meta de los adolescentes y su motivación intrínseca en educación física. Revista de Psicología del Deporte, 15(1), 23-35.
Halbach, A. (2008). Una metodología para la enseñanza bilingüe en la etapa de Primaria Bilingual Methodology in Primary Schools. Revista de Educación, 346, 455-466.

Hernando, A., Hortigüela, D., \& Pérez-Pueyo, Á. (2018). Percepción de coordinadores de programas bilingües y docentes de Educación Física en inglés en secundaria sobre el proceso de implantación y desarrollo del bilingüismo en la Comunidad de Castilla y León. Retos: nuevas tendencias en educación física, deporte y recreación, (33), 63-68.

Hornberger, N. H., \& Link, H. (2012a).Translanguaging and transnational literacies in multilingual classrooms: a biliteracy lens. International Journal of Bilingual Education and Bilingualism, 15(3), 261-278.

Hornberger, N. H., \& Link, H. (2012b). Translanguaging in Today's Classrooms: A Biliteracy Lens. Theory Into Practice(51), 239-247.

Jes, J., \& Molero, J. J. (2009). Aspectos a tener en cuenta en la integración de los contenidos lingüísticos. EmásF: revista digital de Educación Física(9), 6-15.

Khodadady, E., \& Khajavy, G. H. (2013). Exploring the role of anxiety and motivation in foreign language achievement: a structural equation modeling approach. Porta Linguarum(20), 269-286.

Lewis, G., Jones, B., \& Baker, C. (2012).Translanguaging: origins and development from school to street and beyond. Educational Research and Evaluation, 18(7), 641654. doi:10.1080/13803611.2012.718488

Lova, M. (2013). Programas bilingües en Educación Primaria: valoraciones de docentes. Porta Linguarum(20), 253-268.

Lukwu, R. M., \& Guzmán Luján, J. F. (2011). Sport commitment and adherence: A social-cognitive analysis. RICYDE. Revista Internacional de Ciencias del Deporte, 7(25), 277-286.

Macaro, E. (2001). Analysing student teachers' codeswitching in foreign language classrooms: Theories and decision making. The Modern Language Journal, 85(4), 531-548.

Manchunsky, M. (2008). Developing Material for Physical Education lessons in CLIL. Munich: GRIN Verlag.

Marroquín, M. (1984). La escucha activa. Madrid: Asetes.

Molero Clavellinas, J. J. (2009). De la Educación Física hacia la Educación Física bilingüe: un análisis desde el punto de vista metodológico. EmásF: revista digital de Educación Física(10), 7-16.

Molero Clavellinas, J. J. (2011). La planificación de la Educación Física bilingüe: aspectos a tener en cuenta en la integración de los contenidos lingüísticos. EmásF: 
revista digital de Educación Física(9), 6-15.

Moreno-Murcia, J. A., Huéscar, E., Peco, N., Alarcón, E., \& Cervelló, E. (2012). Desarrollo y validación de escalas para la medida de la comunicación en Educación Física y relación con la motivación intrínseca. Universitas Psychologica, 11(3), 957-967.

Moreno-Murcia, J. A., Huéscar, E., Peco, N., Alarcón, E., \& Cervelló, E. (2013). Relación del feed-back y las barreras de comunicación del docente con la motivación intrínseca de estudiantes adolescentes de educación física. Anales de Psicología, 29(1), 257-263. doi:https://doi.org/10.6018/analesps.29.1.161881

Moreno, J. A., González-Cutre, D., \& Chillón, M. (2009). Preliminary validation in Spanish of a scale designed to measure motivation in physical education classes:The perceived locus of causality (PLOC) scale. The Spanish Journal of Psychology, 12(1), 327-337. doi: 10.1017/S1138741600001724

Nishida, T. (1989). A study on standarization of the achievement motivation in physical education test. Japanese Journal of Physical Education(34), 45-65.

Orden 29/2013, de 11 de enero, por la que se modifica la Orden 3331/2010, de 11 de junio, por la que se regulan los institutos bilingües de la Comunidad de Madrid, (2013).

PavónVázquez,V., \& Rubio, F. (2010).Teachers' concerns and uncertainties about the introduction of CLIL programmes. Porta Linguarum(14), 45-58.

Ramos, F., \& Ruiz, J. V. (2010). La Educación Física en centros bilingües. Sevilla: Wanceulen.

Ramos, F., \& Ruiz, J. V. (2011). La Educación Física en centros bilingües de Primaria Inglés-Español: de las singularidades propias del área a la elaboración de propuestas didácticas con AIBLE. RESLA(24), 153170.

Rodríguez-Pérez, N. (2014). Creencias y representaciones de los profesores de lenguas extranjeras sobre la influencia de los factores motivacionales y emocionales en los alumnos y en las alumnas. Porta Linguarum(21), 183-197.

Rotaetxe, K. (1999). Alternancia de código: uso y restricciones tipológicas. Fontes Linguae Vasconum: Studia et Documenta(80), 59-72.

Ruiz Pérez, L. M., Graupera, J. L., Gutiérrez, M., \& Nishida, T. (2004). El Test AMPET de motivación de logro para el aprendizaje en Educación Fïsica: desarrollo y análisis factorial de la versión española. Revista de Educación(335), 195-211.

Ruiz Pérez, L. M., Moreno Murcia, J. A., Ramón Otero, I., \& Alias García, A. (2015). Motivación de lo- gro para aprender en Educación Física: adaptación de la versión española del Test AMPET. Revista Española de Pedagogía(260), 157-175.

Ryan, R. M. (1995). Psychological needs and the facilitation of integrative processes. Journal of Personality, 63(3), 397-427.

Salvador-Garcia, C., Chiva-Bartoll, O., \& Capella-Peris, C. (2020). Bilingual physical education: the effects of CLIL on physical activity levels. International Journal of Bilingual Education and Bilingualism, 1-10.

Salvador-Garcia, C., Chiva-Bartoll, O., \& Gallego, J. (2018). Percepción del alumnado sobre el uso del método AICLE en Educación Física: estudio de caso. Retos, (33), 138-142.

Sánchez-Oliva, D. (2014). Motivación y desarrollo positivo en el alumnado a través de la Educación Física (Tesis doctoral). Universidad de Extremadura.

Sevil, J., Abós, A., Generelo, E., Aibar, A., \& GarcíaGonzález, L. (2016). Importancia del apoyo a las necesidades psicológicas básicas en la predisposición hacia diferentes contenidos en Educación Física. Retos, 29, 3-8.

Thomas, J. R., \& Nelson, J. K. (2007). Métodos de investigación en actividad física. Badalona: Paidotribo.

Torregrosa, M., Sousa, C., Viladrich, C., Villamarín, F., \& Cruz, J. (2008). El clima motivational y el estilo de comunicación del entrenador como predictores del compromiso en futbolistas jóvenes. Psicothema, 20(2), 254-259.

Viciana, J., Cervelló, E. M., \& Ramírez-Lechuga, J. (2007). Effect of manipulating positive and negative feedback on goal orientations, perceived motivational climate, satisfaction, task choice, perception of ability, and attitude toward physical education lessons. Perceptual and motor skills, 105(1), 67-82. doi:10.2466/ pms.105.1.67-82

Viciana, J., Salinas, F., Ramírez, J., Cocca, A., \& Cervelló, E. (2007). Efectos del feedback afectivo sobre el aprendizaje motor y la satisfacción en educación física. Revista Mexicana de Psicología, 394-395.

\section{(Footnotes)}

${ }^{1}$ Hace referencia al individuo sin distinción de género (alumna o alumno)

${ }^{2}$ Hace referencia al individuo sin distinción de género (profesora o profesor) 